\title{
Evidence of Chaos in Indoor Pedaling Motion using Non-linear Methods
}

\author{
Juan-Carlos Quintana-Duque and Dietmar Saupe
}

\subsection{INTRODUCTION}

Quantitative studies of human motion have typically focused on properties of the average motion and their fluctuations were considered noise. Recent publications (for example (Davids, et al., 2006)) found evidence that the alleged noise, called motion variability, contains important information useful to characterize mature motor skills and healthy states. Motion variability is always present in healthy human beings and even Olympic athletes show motion variability despite of several years of intensive training of specific movements. Lack or excess of variability are now understood as a deficiency in motor skills.

Recently, non-linear methods for analysis of time series have been applied to quantify the amount of motion variability. In that approach a dynamical system is presumed such that time series can be considered as noisy measurements of some function of particular solutions to the system. Dynamic invariants characterize properties of the solution space of the corresponding unknown dynamical systems.

Dynamic invariants can be calculated from trajectories in state space of a dynamical system. Only in rare cases, when a very simple dynamical system is given explicitly, it may be possible to rigorously compute dynamic invariants. Thus, in practice only a numerical approximation is feasible. Moreover, as mentioned above, for many applications, and in particular for all practical applications in sport science, the underlying dynamical system is assumed to model a very complex system like the human body performing a certain task. In this case the dynamical system is not known explicitly. Even if it was, it would typically contain a myriad of variables defying any mathematical or even numerical analysis.

However, since the discovery of Takens (1981) of a topologically faithful reconstruction of state space of dynamical systems in a lower dimensional so called embedding space, it became possible to estimate dynamic invariants only from the aforementioned noisy measurements of some function or projection of a trajectory in the original (unknown) state space. For example, in sport science applications one does not have access to all neural and muscular signals of the human body (i.e., the state space) or complete knowledge of their governing dynamical system to directly calculate the dynamic properties of the human motion control system, but one can record the resulting human motion using video analysis or direct motion capturing. In the case of cyclical motion of an athlete on an ergometer that 
we consider in this paper, we propose to measure the three-dimensional spatial position of one of the knee joints which serve as observations of the underlying complex dynamical system. The time series of one component of the coordinate position (e.g., the $X$-coordinate) may be enough to reconstruct the dynamic behavior of the human motion system that is to be quantified by dynamic invariants.

The dynamic invariants calculated from a time series are meaningful only when there is indeed deterministic nonlinearity in the time series. However, time series data can be anything between purely random and strictly deterministic. In order to test the presence of deterministic nonlinearity in a time series, it has been suggested to calculate the maximal Lyapunov exponent (this dynamic invariant will be explained in following) and to use the surrogate data methodology.

A positive maximal Lyapunov exponent is a characteristic of chaotic time series but this exponent alone cannot be used for proving strictly deterministic chaos because random data may also produce a positive maximal Lyapunov exponent. Furthermore, the surrogate data methodology is used to test a given time series for membership of specific class of dynamical systems comparing features of the original data and certain surrogate data which can be generated algorithmically from the original data. In order to complement the evidence of chaos, the surrogate data must keep most of the original linear characteristics of the original data while making the original chaotic characteristics random. If the result of the surrogate data test is negative and there is a positive maximal Lyapunov exponent within time series, one can conclude that the time series data come from a nonlinear chaotic dynamical system.

Previous works on gait analysis (e.g., (Nessler, et al., 2009) and (Stergiou, 2004)) presented results confirming the hypothesis that gait motion data come from a deterministic chaotic system. This conclusion was reached comparing original with surrogate data created by a method operating in the frequency domain (i.e., phase randomization). However, this method is inappropriate for original data exhibiting strong cyclic components because it destroys the essential periodic original features. For such kind of cyclical motion data it is more natural to ask if there can be demonstrated determinism for durations longer than the data cycle length (Miller, et al., 2006). Thus, the reported evidence of deterministic chaos on gait motion data using the surrogate data obtained from randomizing the phase in the frequency domain, might be incomplete. The chaotic relation between cycles was not tested. The finding was only that the gait motion data is not random, which is obvious. In the case of gait motion (and in general for any cyclical motion), one needs to validate the hypothesis that intercycle fluctuations are deterministic chaos and not random noise.

Knowing this, we propose to analyze whether intercycle dynamics of indoor pedaling motion come from a deterministic chaotic system using the pseudo periodic surrogate (PPS) method described in (Small, et al., 2001). The PPS method creates surrogate data from original time series data preserving the original intracycle dynamics (dynamic patterns within one period of a cyclic pattern) while changing the original intercycle dynamics (dynamic patterns between different periods across a cyclic pattern). 
As far as we know, there is no previous work about evidence of chaos in pedaling motion. Here we analyzed the time series of the angle and the $\mathrm{X}$ coordinate position of the knee joint recorded during pedaling under different constraints (low and high cadence with low and high pedal brake force). In order to find evidence of chaos within pedaling motion data, we calculated the maximal Lyapunov exponent. Additionally we used the surrogate methodology with correlation dimension as test statistic and with surrogate data created by methods based on the frequency domain and the embedding space. We found that our recorded motion data of indoor pedaling have intercycle chaotic dynamics.

\subsubsection{Dynamical systems and dynamic invariants}

A dynamical system consists of the dynamics taking place in a state space. The state space usually is a linear space of all possible world-states of the system in question. Each world state represents a complete snapshot of the system at some moment in time. In the discrete-time case, the dynamics is given by a function that transforms one point in state space (world-state), representing the state of the system "now", into another point representing the state of the system one time unit "later" (i.e., a second world-state). Thus, if points in state space are denoted by vectors $\mathbf{x}_{\mathrm{n}}$ of $m$ components and the dynamics are given by a function $\mathrm{f}$ we have

$$
\mathbf{x}_{\mathrm{n}+1}=\mathrm{f}\left(\mathbf{x}_{\mathrm{n}}\right), \mathbf{x}_{\mathrm{n}}=\left[x_{n, 1}, x_{n, 2}, x_{n, 3}, \ldots, x_{n, m}\right] \text {. }
$$

Once an initial world-state is chosen, the dynamics determines the world-state at all future times forming a trajectory in state space.

In real-world applications, usually we do not have complete knowledge, let alone access to the $m$-dimensional state space but we have to work with one or several one-dimensional observations $y_{n}$. Takens' theorem, described in (Takens, 1981), guarantees that the observations $y_{n}$ will be enough to reconstruct the attractor of the dynamical system in the embedding space with dimension $d_{e}$ if the observations are infinite and without noise. In the practice, a sequence of observations is enough to approximate the attractor when the data is sufficiently large and contains low levels of noise. The embedding is defined by equation (30.2) with the embedding dimension $d_{e}$ and the embedding delay $\tau$ as parameters, where the dimension $\mathrm{d}_{\mathrm{e}}$ of the embedding space must be larger than twice the box counting dimension of the attractor plus one. See more details in (Eckmann, et al., 1985).

$$
\mathbf{v}_{\mathrm{n}}=\left(\mathrm{y}_{\mathrm{n}}, \mathrm{y}_{\mathrm{n}-\tau} \ldots, \mathrm{y}_{\mathrm{n}-\left(\mathrm{d}_{\mathrm{e}}-1\right) \tau}\right)
$$

The accuracy of numerical estimation of invariants from experimental data depends on the quantity and quality of the time series data as well as on the quality of the reconstruction of the complexity of the dynamical system using an embedding space. 


\subsubsection{Dynamic invariants: maximal Lyapunov exponent}

Lyapunov exponents describe the average expansion respectively contraction of the evolution of trajectories on an attractor of a dynamical system. The most robust and important one is the maximal Lyapunov exponent $\lambda$. This dynamic invariant is calculated from a time series using embedding points defined by equation (30.2).

Let $\mathbf{v}_{n_{0}}$ and $\mathbf{v}_{n_{1}}$ be two points in the embedding space with a sufficiently small Euclidian distance $\delta_{0}=\left\|\mathbf{v}_{n_{0}}-\mathbf{v}_{n_{1}}\right\|$ and denote by $\delta_{\Delta \mathrm{n}}$ the distance between the trajectories emerging from these points at some time $\Delta \mathrm{n}$ ahead, $\delta_{\Delta \mathrm{n}}=$ $\left\|\mathbf{v}_{n_{0}+\Delta \mathrm{n}}-\mathbf{v}_{n_{1}+\Delta \mathrm{n}}\right\|$. Then, if $\delta_{\Delta \mathrm{n}} \approx \delta_{0} \mathrm{e}^{\lambda \cdot \Delta \mathrm{n}}$ for a range of values $\Delta \mathrm{n}$ and in expectation for points $\mathbf{v}_{\mathrm{n} 0}$ on the attractor the maximal Lyapunov exponent is $\lambda$. If $\lambda$ is positive, there is a strong signature of chaos in the time series data. The higher the instability and the divergence of neighboring trajectories in embedding space, the larger is the value of $\lambda$.

Numerically, the maximal Lyapunov exponent $\lambda$ is calculated as the slope of the average logarithmic divergence of the neighboring trajectories in the reconstructed attractor in the embedding space. The algorithm for calculating $\lambda$ is the following: A reference point $\mathbf{v}_{n_{0}}$ is chosen and its nearest neighbors $\mathbf{v}_{n_{1}, n_{2}, \ldots}$ with distance to the reference point less than $\mathrm{r}$ are selected from the reference point's neighborhood $\mathfrak{U}$. Then, one computes the distances of all selected neighbors to the reference point following the trajectories as a function of the relative time $\Delta \mathrm{n}$ for $0 \leq \Delta \mathrm{n} \leq \Delta \mathrm{n}_{\max }$. Repeating the latter for many (N) reference points $\mathbf{v}_{n_{0}}$ and calculating the averages $S(\Delta \mathrm{n})$ of these results, see (30.3), noise in the data and fluctuations of the effective divergence will average out. If for some range of $\Delta \mathrm{n}$ the function $S(\Delta \mathrm{n})$ exhibits a robust linear increase, its slope is a stable and robust estimate of the maximal Lyapunov exponent. See more details in (Kantz and Schreiber, 2004).

$$
\mathrm{S}(\Delta n)=\frac{1}{\mathrm{~N}} \sum_{\mathrm{n}_{0}=1}^{\mathrm{N}} \ln \left(\frac{1}{\left|\mathfrak{H}\left(\mathbf{v}_{n_{0}}\right)\right|} \sum_{\mathbf{v}_{n} \in \mathfrak{2}\left(\mathbf{v}_{n_{0}}\right)}\left\|\mathbf{v}_{n_{0}+\Delta n}-\mathbf{v}_{n+\Delta n}\right\|\right)
$$

\subsubsection{Dynamic invariants: correlation dimension}

The correlation dimension $d_{c}$ is a topological property of an invariant set (attractor) of a dynamical system. One considers the probability that two points chosen randomly from the invariant set, and according to the dynamics of the system, will be within distance $\varepsilon$ of each other. Then one examines how this probability changes as the distance $\varepsilon$ varies. If the probability scales as $\varepsilon^{d_{c}}$ then the exponent $d_{c}$ is called correlation dimension.

The correlation dimension can be numerically computed from a long trajectory as follows: One calculates the correlation sum $\mathrm{C}(\mathrm{N}, \varepsilon)$, equation (30.4), which approximates the desired probability. This sum is the relative number of 
pairs of embedding points $\left(\mathbf{v}_{\mathbf{i}}, \mathbf{v}_{\mathbf{j}}\right)$ with distance less than $\varepsilon$ of each other. By definition, equation (30.5), as the data size grows, $N \rightarrow \infty$, and for small $\varepsilon \rightarrow 0$, one expects that correlation sum scales with a power law $\mathrm{C}(\mathrm{N}, \varepsilon) \propto \varepsilon^{d_{c}}$. In practice, one inspects the function $\ln \mathrm{C}(\mathrm{N}, \varepsilon)$ versus $\ln \varepsilon$ for a linear region. If the linear region exists, the slope of this region is an approximation of the correlation dimension $d_{c}$.

In order to avoid the influence of temporal data correlation in the correlation sum $\mathrm{C}$, one excludes those pairs of embedding points which are close not because the geometry of the attractor but just because they are closer than $n_{\min }$ in (discrete) time. The time window $n_{\min }$ is called Theiler window. Furthermore, the formula in equation (30.4) is defined using the Heaviside step function $\Theta$, which is $\Theta(\mathrm{x})=$ 0 for $\mathrm{x} \leq 0$ and $\Theta(\mathrm{x})=1$ for $\mathrm{x}>0$. See more details in (Kantz, et al., 2004).

$$
\begin{gathered}
\mathrm{C}(\mathrm{N}, \varepsilon)=\frac{2}{\left(\mathrm{~N}-n_{\min }\right)\left(\mathrm{N}-n_{\min }-1\right)} \sum_{\mathrm{i}=1}^{\mathrm{N}} \sum_{\mathrm{j}=\mathrm{i}+n_{\min }}^{\mathrm{N}} \Theta\left(\varepsilon-\left\|\mathbf{v}_{\mathrm{i}}-\mathbf{v}_{\mathrm{j}}\right\|\right) \\
d_{c}=\lim _{\varepsilon \rightarrow 0} \lim _{\mathrm{N} \rightarrow \infty} \frac{\partial \log (\mathrm{C}(\mathrm{N}, \varepsilon))}{\partial \log \varepsilon}
\end{gathered}
$$

However, locating the linear part, i.e., the scaling region, of the function $\log C(N, \varepsilon)$ versus $\log \varepsilon$ is often subjective. A modification for the calculation of correlation dimension proposed by Judd in (Judd, 1992) aims at reducing such effects by modeling the growth of correlation sum as

$$
\mathrm{C}(\mathrm{N}, \varepsilon) \propto \varepsilon^{d_{c}} \mathrm{p}(\varepsilon)
$$

where $\mathrm{p}(\varepsilon)$ is a (e.g., quadratic) polynomial. This has an interesting benefit. For many natural objects the dimension is not the same at all length scales. If one observes a large river stone its surface at its largest length scale is very nearly twodimensional, but at smaller length scales one can discern the details of grains which add to the complexity and increase the dimension at smaller scales. Consequently, it is natural to consider dimension $d_{c}$ as a function of $\varepsilon$ and write $d_{c}(\varepsilon)$ (Small, et al., 2001). The scale dependent correlation dimension $d_{c}(\varepsilon)$ and the coefficients of the polynomial can be estimated together by unconstrained nonlinear optimization using correlations sums $C\left(N, \varepsilon^{\prime}\right)$ in a suitable chosen scaling range near $\varepsilon$.

\subsubsection{Surrogate data}

Surrogate data are new time series created from original time series data keeping some of the original linear characteristics and making the original non-linear 
characteristics random. The choice of method to generate surrogate data depends on the null hypothesis that one wants to test, comparing features of the original and surrogate data. The methods can be based on the original time series, on the data in the frequency domain or in the embedding space. In this paper we used the last two.
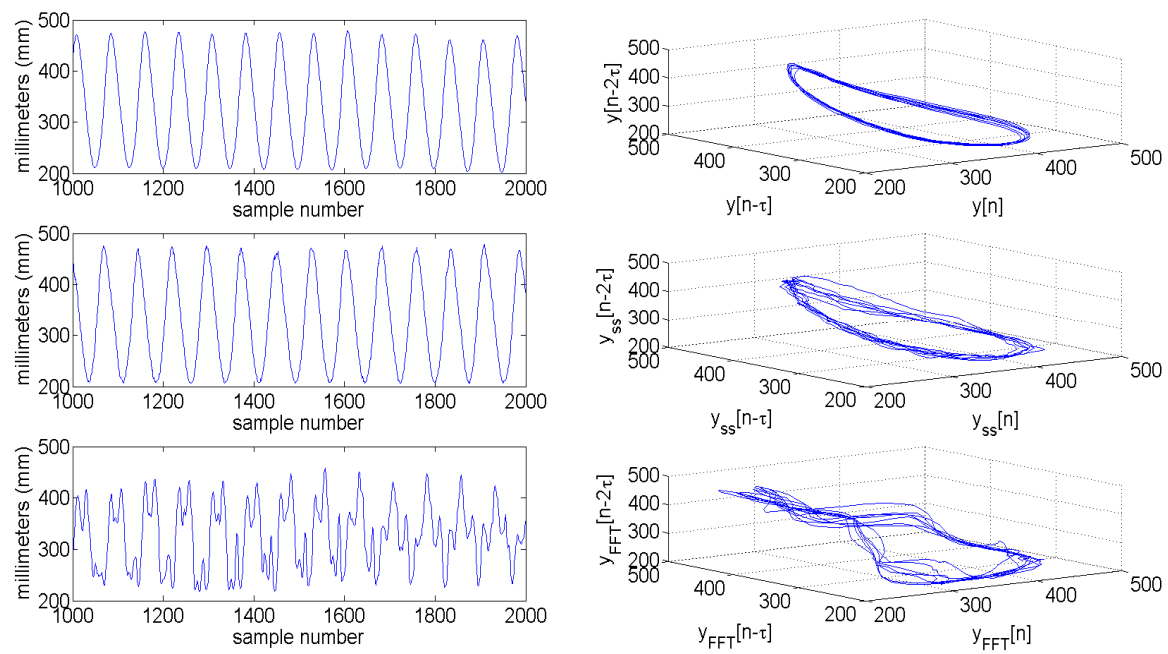

Figure 30.1 Effects of different surrogate methods on time and in embedding space. Top: Original data; middle: Surrogate data based on embedding; bottom: Surrogate data based on FFT. We plotted the time series (left) and the corresponding embedding space (right). The embedding is with dimension $\mathrm{d}_{\mathrm{e}}=3$ and delay parameter $\tau=15$. The surrogate method based on embedding keeps the intracycle structure of original data (see the similarity between original and surrogate time series) while destroying the intercycle structure (see the noisy attractor in embedding space). The surrogate method based on FFT destroys both the intracycle and the intercycle structure (the attractor in embedding space has a different shape).

The method to create surrogate data based on embedding space called pseudo periodic surrogate algorithm PPS, proposed in (Small, et al., 2002), is used to test the null hypothesis that the original time series data, exhibiting pseudo-periodic dynamics, come from a dynamical system with an attractor whose periodic orbit is driven by uncorrelated noise. For this algorithm, the attractor of the observed dynamical system is first reconstructed from the time series data $y_{n}$ using an embedding space defined by equation (30.2). After that, the embedding points of neighboring trajectories in the reconstructed attractor are used to create a new attractor with noisy trajectories. That is, one selects an initial embedded point $\mathbf{v}_{\mathbf{i}}$ from the reconstructed attractor as start point of the surrogate trajectory. For the following point in time of the surrogate trajectory, one of the embedding neighbors $\mathbf{z}_{\mathrm{i}}$ of $\mathbf{v}_{\mathrm{i}}$ is randomly selected with a probability depending on the noise level parameter $\rho$, given by equation 


$$
\operatorname{Prob}\left(\mathbf{v}_{\mathrm{i}}=\mathbf{z}_{\mathrm{i}}\right) \propto \exp \left(-\left\|\mathbf{z}_{\mathrm{i}}-\mathbf{v}_{\mathrm{i}}\right\| / \rho\right) .
$$

The following point in time $\mathbf{z}_{\mathbf{i}+1}$ of the selected embedding point $\mathbf{z}_{\mathbf{i}}$ is selected as the next point of the surrogate sequence being created. The last step is repeated for last selected embedding point until the number of desired points is achieved creating in this way a noisy attractor. Finally, the new surrogate time series data is constructed from the embedding points of the noisy attractor. With an appropriate choice of the embedding parameters $\left(\tau\right.$ and $\left.d_{e}\right)$ and the noise parameter $\rho$, intracycle dynamics are preserved but intercycle dynamics are not (see Figure 30.1 middle). If $\rho$ is large, then the points are simply temporally uncorrelated random points. If $\rho$ is too small then the surrogate and original are identical. See more details about how to select a suitable value of $\rho$ in (Small, et al., 2002).

The method to created surrogate data based on frequency space, proposed in (Theiler, 1992), is used to test the null hypothesis that the original time series data correspond to linearly filtered noise. One generates phase-randomized surrogates from the time series data $\mathrm{y}_{\mathrm{n}}$ by computing Fast Fourier Transforms (FFT), randomizing the phase spectra, and finally computing the inverse FFT. With this method, the power spectrum and correlation function are preserved from the original time series while the probability distribution of the values is changed and the intracycle dynamics is destroyed (see Figure 30.1 bottom).

\subsection{METHODS}

The pedaling motion of a healthy rider without muscle fatigue was recorded with a sampling rate of $100 \mathrm{~Hz}$ using a motion capture system (LUKOtronic) and a configurable cycling ergometer (Cyclus2). Four tests of two minutes each were made with several cadences ( 80 and $100 \mathrm{rpm}$ ) and pedal brake forces (120 and 140 Newton). The dynamic invariants were calculated from the time series data of the $X$-coordinate of the knee joint position and the knee joint angle (formed by three markers attached on knee, ankle, and hip joints). Raw data was used for the reconstruction of the attractor in embedding space. The calculation of the embedding parameters and the maximal Lyapunov exponent was done using the TISEAN software described in (Hegger, et al., 1999) and the surrogate data analysis was done using the ANTA software described in (Small, 2005).

The calculation of the embedding parameters $d_{e}$ and $\tau$ was done separately for each time series. The first zero-crossing of the autocorrelation was used as embedding delay $\tau$, and in order to choose the embedding dimension $\mathrm{d}_{\mathrm{e}}$, the false neighbor algorithm (FNN) was applied with the previously calculated embedding delay $\tau$ as parameter. See (Kantz, et al., 2004) for a description of the used methods.

The hypothesis, that intercycle irregularities of pedaling motion data are most likely due to deterministic chaos rather than random inputs, was analyzed using the graphical method proposed in (Small, 2002). This method consists in a graphical 
comparison between the correlation dimension values of 50 different surrogate time series and the correlation dimension value of the original time series. The contour plot in each panel in Figure 30.2 is a representation of the probability distribution function (PDF) of scale dependent the correlation dimension $\mathrm{d}_{\mathrm{c}}(\varepsilon)$ of surrogate data for distinct values of $\varepsilon$. Each vertical slice (i.e., for a given $\varepsilon$ ) corresponds to a single PDF calculated using the density kernel estimation algorithm (Silverman, 1986) with a Gaussian kernel. The isocline lines of the contour plot are selected automatically and uniformly. Points outside the outermost isocline correspond to correlation dimension values that are outside the range of dimensions for the surrogate data.

For the calculation of the maximal Lyapunov exponent from equation (30.3) we used a neighborhood $\mathfrak{A}\left(\mathbf{v}_{\mathrm{n}_{0}}\right)$ of a size $\sigma\left(\mathrm{y}_{\mathrm{n}}\right) / 100$, with $\sigma\left(\mathrm{y}_{\mathrm{n}}\right)$ as standard deviation of the discrete time series $y_{n}$. In order to get reliable results of this invariant for each time series, we calculated averages of the maximal Lyapunov exponent values obtained from embedding spaces created using embedding dimensions $\mathrm{d}_{\mathrm{e}}, \mathrm{d}_{\mathrm{e}}+1, \mathrm{~d}_{\mathrm{e}}+2, \mathrm{~d}_{\mathrm{e}}+3$, and $\mathrm{d}_{\mathrm{e}}+4$.

\subsection{RESULTS}

Table 30.1 shows the embedding parameters (embedding dimension $\mathrm{d}_{\mathrm{e}}$ and embedding delay $\tau$ ), and the maximal Lyapunov exponent $\kappa$ calculated for all four tests. Pedal brake force is given in Newton $(\mathrm{N})$, the cadence in revolutions per minute (rpm) and the power in Watt (W). The maximal Lyapunov units are given in $1 / s$.

Table 30.1 Calculated embedding dimension $\boldsymbol{d}_{\boldsymbol{e}}$, embedding delay $\boldsymbol{\tau}$, and maximal Lyapunov exponent $\kappa$ of time series of knee joint angle and knee joint position

\begin{tabular}{|c|c|c|c|c|c|c|c|c|c|c|c|c|}
\hline & \multicolumn{3}{|c|}{$\begin{array}{c}140 \mathrm{~N} 100 \mathrm{rpm} \\
(218 \mathrm{~W})\end{array}$} & \multicolumn{3}{|c|}{$\begin{array}{c}120 \mathrm{~N} 100 \mathrm{rpm} \\
(187 \mathrm{~W})\end{array}$} & \multicolumn{3}{|c|}{$\begin{array}{c}140 \mathrm{~N} 80 \mathrm{rpm} \\
(176 \mathrm{~W})\end{array}$} & \multicolumn{3}{|c|}{$\begin{array}{c}120 \mathrm{~N} 80 \mathrm{rpm} \\
(150 \mathrm{~W})\end{array}$} \\
\hline & $\mathrm{d}_{\mathrm{e}}$ & $\tau$ & $\Lambda$ & $\mathrm{d}_{\mathrm{e}}$ & $\tau$ & $\kappa$ & $\mathrm{d}_{\mathrm{e}}$ & $\tau$ & $\kappa$ & $\mathrm{d}_{\mathrm{e}}$ & $\tau$ & $\kappa$ \\
\hline Knee angle & 4 & 23 & $\begin{array}{c}0.2211 \\
\pm 0.0223\end{array}$ & 4 & 22 & $\begin{array}{c}0.1872 \\
\pm 0.0279\end{array}$ & 4 & 23 & $\begin{array}{c}0.211 \\
\pm 0.0393\end{array}$ & 4 & 33 & $\begin{array}{c}0.32 \\
\pm 0.0240\end{array}$ \\
\hline Knee $X$-coordinate & 4 & 18 & $\begin{array}{c}0.1849 \\
\pm 0.0094\end{array}$ & 4 & 21 & $\begin{array}{c}0.2373 \\
\pm 0.0357\end{array}$ & 4 & 21 & $\begin{array}{c}0.1999 \\
\pm 0.0195\end{array}$ & 4 & 25 & $\begin{array}{c}0.2607 \\
\pm 0.0254\end{array}$ \\
\hline
\end{tabular}

In Figure 30.2, we show the results of graphical method proposed in (Small, 2002) to compare correlation dimension values between original data and surrogate data. We used two different methods to create surrogate data based on frequency domain and embedding space. Additionally, in order to check whether a correlation between power and correlation dimension exists, we plot the correlation dimension for all test in

Figure 303. 

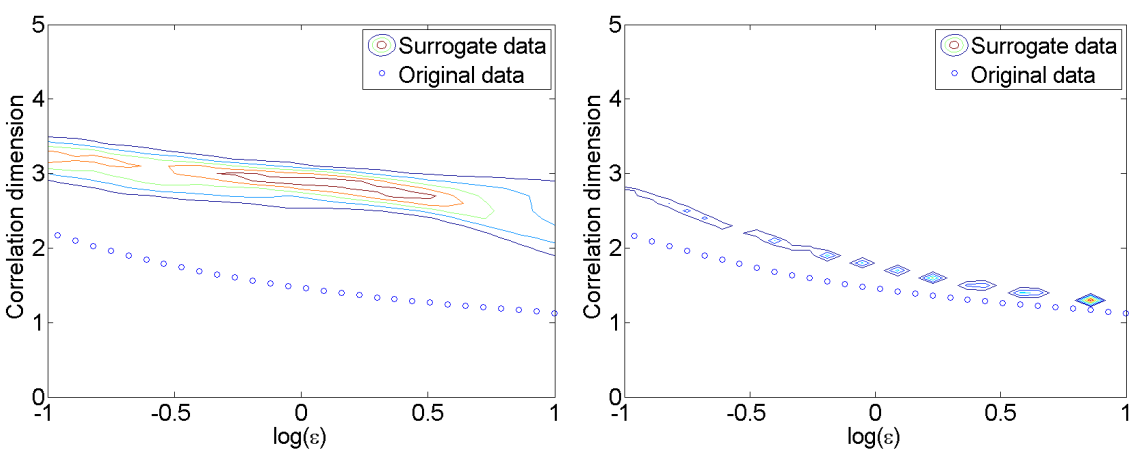

Figure 30.2 Results obtained from 50 surrogate data sets created with a method based on frequency domain (left) and on embedding space (right) for the time series data of the test $140 \mathrm{~N} 100 \mathrm{rpm}$
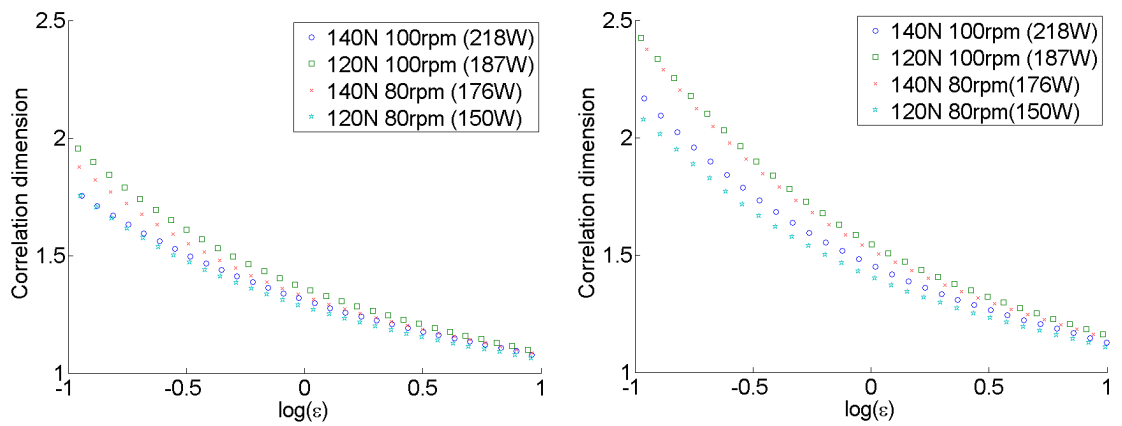

Figure 30.3 Comparison of correlation dimension values between all tests using knee joint angle data (left) and $\boldsymbol{X}$-coordinate of knee joint position data (right). There is no evidence of a linear relation between the value of invariants and workload intensity

\subsection{CONCLUSIONS AND FUTURE WORK}

Evidence of deterministic chaos was found in all time-series of knee motion data recorded during indoor pedaling following the methodology suggested in (Small, et al., 2002). This methodology consists on the search of evidence of chaos within time series based on the calculation of the maximal Lyapunov exponent and on a test of non-linearity using surrogate data with the correlation dimension $d_{c}(\varepsilon)$ as test statistic.

Table 30.1 shows that the time series of all tests done with different workloads have positive maximal Lyapunov exponents $\Lambda$, which is a characteristic of chaotic dynamical systems. In addition to this, analysis using surrogate data (e.g., Figure 30.2) was done in order to test two different null hypotheses: (1) the time series data correspond to linearly filtered noise (i.e., surrogate data were created base on frequency domain method) and (2) that the data come from a 
dynamical system with an attractor whose periodic orbit is driven by uncorrelated noise (i.e., surrogate data were based on embedding space method). For both null hypotheses, we found clear differences between the surrogate data and original data suggesting that any long term determinism of period longer than the data cycle length exists within time series data, and that the pedaling motion comes from a deterministic chaotic system. In general, these differences were more evident for the surrogate data created using frequency method than from that using embedding space (see Figure 30.2). Evidence of chaos in other cyclic human motion as gait data was reported in (Dingwell, 2000) and in (Miller, et al., 2006) but as far as we know, there are no previous works about evidence of chaos in pedaling motion.

Furthermore, there is no evidence of a linear relation between the value of invariants and workload intensity with speed constraints (see Table 30.1 and

Figure 30.3). Similar conclusions have been reported before in literature about gait data and speed constraints, for example in (Buzzi and Ulrich, 2004).

Our future research will focus on (1) the effects on dynamic invariants due to external (i.e., cadence and workload) and internal constraints (i.e., fatigue and muscle training), and (2) which invariants are more suitable for analysis according to the characteristics of pedaling motion.

\subsection{ACKNOWLEDGEMENTS}

This work was supported by the DFG Research Training Group 1042 Explorative Analysis and Visualization of Large Information Spaces at the University of Konstanz.

\subsection{REFERENCES}

Broer, H. and Takens, F., 2010, Dynamical Systems and Chaos. (Groningen: Springer Verlag).

Buzzi, U.H. and Ulrich, B.D., 2004, Dynamic stability of gait cycles as a function of speed and system constraints. Motor Control, 8(3). p. 241.

Davids, K., Bennett, S. and Newell, K., 2006, Movement system variability. (Champaign: Human Kinetics Publishers).

Dingwell, J.B. and Cusamano, J.P., 2000, Nonlinear time series analysis of normal and pathological human walking. Chaos: An Interdisciplinary Journal of Nonlinear Science, 10, pp. 848-863.

Eckmann, J. and Ruelle, D., 1985, Ergodic theory of chaos and strange attractors. Reviews of modern physics, 57(3), p. 617.

Harbourne, R.T., and Stergiou, N., 2009, Movement variability and the use of nonlinear tools: principles to guide physical therapist practice. Physical Therapy, 89(3), pp. 267-282.

Hegger, R., Kantz H. and Schreiber, T., 1999, Practical implementation of nonlinear time series methods: The TISEAN package, Chaos: An Interdisciplinary Journal of Nonlinear Science, 9(2), pp. 413-435. 
Judd, K., 1992, An improved estimator of dimension and some comments on providing confidence intervals. Physica D: Nonlinear Phenomena, 56(2-3), pp. 216-228.

Kantz, H. and Schreiber, T., 2004, Nonlinear time series analysis. (Cambridge University Press).

Miller, D., Stergiou, N. and Kurz, M., 2006, An improved surrogate method for detecting the presence of chaos in gait. Journal of Biomechanics, 39(15), pp. 2873-2876.

Nessler, J., Leone, C.D. and Gilliland, S., 2009, Nonlinear time series analysis of knee and ankle kinematics during side by side treadmill walking. Chaos: An Interdisciplinary Journal of Nonlinear Science, 19(2), pp. 26104-26104.

Small, M., 2005, Applied nonlinear time series analysis: applications in physics, physiology and finance. (London: World Scientific Pub Co Inc).

Small, M., Judd, K. and Mees, A., 2001, Testing time series for nonlinearity. Statistics and Computing, 11(3), pp. 257-268.

Small, M. and Tse, C., 2002, Applying the method of surrogate data to cyclic time series. Physica D: Nonlinear Phenomena, 164(3-4), pp. 187-201.

Silverman, B.W., 1986, Density Estimation for Statistics and Data Analysis (London: Chapman \& Hall).

Stergiou, N., Harbourne, R. and Cavanaugh, J., 2006, Optimal movement variability: a new theoretical perspective for neurologic physical therapy. Journal of Neurologic Physical Therapy, 30(3), p. 120.

Takens, F., 1981, Detecting strange attractors in turbulence. Dynamical Systems and Turbulence, 898(1), pp. 366-381.

Theiler, J. et al., 1992, Testing for nonlinearity in time series: the method of surrogate data. Physica D: Nonlinear Phenomena, 58(1), pp. 77-94.

Wolf, A., et al., 1985, Determining Lyapunov exponents from a time series. Physica D: Nonlinear Phenomena, 16(3), pp. 285-317.

Zhongua, L. 2010, Chaotic time series analysis. Mathematical problems in Engineering, 2010(1), p. 31. 